Applied Mathematical Sciences, Vol. 7, 2013, no. 122, 6095 - 6099

HIKARI Ltd, www.m-hikari.com

http://dx.doi.org/10.12988/ams.2013.310564

\title{
Purely Finitely Additive Measures as Generalized Elements in a Maximin Problem
}

\author{
Artem Baklanov \\ Ural Federal University \\ 620002, Russia, Ekaterinburg, ul. Mira, 19 \\ artem.baklanov@gmail.com
}

Copyright (C) 2013 Artem Baklanov. This is an open access article distributed under the Creative Commons Attribution License, which permits unrestricted use, distribution, and reproduction in any medium, provided the original work is properly cited.

\begin{abstract}
We study the asymptotic behavior of maximin values of a payoff function, when admissible controls tend to infinity. The payoff function is superposition of a continuos function and a function that is uniform limit of step functions. An extension in the class of finitely additive measures is used.
\end{abstract}

Mathematics Subject Classification: 46N10, 49J35, 49J45, 49N99

Keywords: purely finitely additive measures, extension, maximin.

It is natural in control problems to use extensions constructions due to the non-existence of optimal solutions [1]. Moreover, extensions are needed for a 'regularization' of practically interesting problems that are associated with asymptotic relaxations of constraints (see $[2,3]$ ). Obviously, game problems require extensions [4]. These problems can be divided into two types: those for which it is possible to well define an extension only on the product of ordinary controls sets of players [4-6], and those for which it is sufficient to construct an extension of an ordinary controls set for each player separately [7-10]. If we deals with a maximin problem of the second type, then we can use the following representation of the maximin value $V_{\text {ext }}$ after an extension:

$$
V_{e x t}=\max _{\nu \in B} \min _{\mu \in A} \tilde{\alpha}(\mu, \nu),
$$


where $A, B$ are some sets of generalized elements (controls) and $\tilde{\alpha}$ is the generalized payoff function. In [7-10] the extension in the class of finitely additive measures (FAM) was used. In these papers sets $A$ and $B$ were some compacta in $*$-weak topology. The using of FAM helps to deal with the case of discontinuous control coefficients in the right-hand part of a differential equation. Often in this case purely FAM are essential elements of sets $A, B$ (see $[7,10]$ ). In this paper we consider a maximin problems such that admissible controls of players in some sense tends to infinity. We study asymptotics of values of the problems. We show that in this case sets $A, B$ are subsets of purely FAM. The present paper extends results of [11].

Let $\left(X, \rho_{X}\right)$ and $\left(Y, \rho_{Y}\right)$ be unbounded metric spaces; fix $x_{0} \in X$ and $y_{0} \in Y$. By $S_{X}^{\varepsilon}$ we denote open $\varepsilon$-neighborhood of $x_{0}$ w.r.t. $\rho_{X}$ and by $S_{Y}^{\kappa}$ we denote open $\kappa$-neighborhood of $y_{0}$ w.r.t. $\rho_{Y}$; here $\left.\varepsilon \in\right] 0, \infty[, \kappa \in] 0, \infty[$. Let $H_{X}^{\varepsilon} \triangleq X \backslash S_{X}^{\varepsilon}, H_{Y}^{\kappa} \triangleq Y \backslash S_{Y}^{\kappa}$. By definition, put $\mathcal{H}_{X} \triangleq\left\{H_{X}^{\varepsilon}: \varepsilon \in\right] 0, \infty[\}, \mathcal{H}_{Y} \triangleq$ $\left\{H_{Y}^{\kappa}: \kappa \in\right] 0, \infty[\}$. Families $\mathcal{H}_{X}$ and $\mathcal{H}_{Y}$ are filter bases in $X$ and $Y$ respectively [12]. By $\mathcal{L}_{X}$ and $\mathcal{L}_{Y}$ we denote semialgebras of subsets of $X$ and $Y$ respectively such that: $\left(\forall x_{0} \in X \forall \varepsilon \in\right] 0, \infty\left[: S_{X}^{\varepsilon} \in \mathcal{L}_{X}\right) \&\left(\forall y_{0} \in Y \forall \kappa \in\right] 0, \infty\left[: S_{Y}^{\kappa} \in \mathcal{L}_{Y}\right)$.

By $\mathbb{R}^{n}$ we denote $n$-dimensional arithmetic space. Let $\mathbf{u}: X \rightarrow \mathbb{R}^{k}$ and $\mathbf{v}: Y \rightarrow \mathbb{R}^{l}$ be uniform limits of $\mathcal{L}_{X^{-}}$-step and $\mathcal{L}_{Y^{-}}$-step functions respectively. Let $\Upsilon, \Upsilon: \mathbb{R}^{k} \times \mathbb{R}^{l} \rightarrow \mathbb{R}$, be a jointly continuous payoff function. Thus,

$$
\Upsilon(\mathbf{u}(x), \mathbf{v}(y)) \in \mathbb{R} \forall \varepsilon \in] 0, \infty\left[\forall x \in H_{X}^{\varepsilon} \forall \kappa \in\right] 0, \infty\left[\forall y \in H_{Y}^{\kappa} .\right.
$$

Now we can consider the following maximin problem for some $\varepsilon \in] 0, \infty[, \kappa \in$ ]0, $\infty$ [. The first player minimizes value of $\Upsilon$ by choosing $x \in H_{X}^{\varepsilon}$, the second player maximizes value of $\Upsilon$ by choosing $y \in H_{Y}^{\kappa}$. Thus we deal with problems

$$
\left.\Upsilon(\mathbf{u}(x), \mathbf{v}(y)) \rightarrow \sup _{y \in H_{Y}^{\kappa}} \inf _{x \in H_{X}^{\varepsilon}}, \quad \varepsilon \in\right] 0, \infty[, \kappa \in] 0, \infty[
$$

We will investigate asymptotics of values $(2)$ as $\varepsilon, \kappa \rightarrow \infty$. Note that this asymptotics do not depend on $x_{0}$ and $y_{0}$.

By definition, put $\mathrm{V}(\varepsilon, \delta) \triangleq \sup _{y \in H_{Y}^{\kappa}} \inf _{x \in H_{X}^{\varepsilon}} \Upsilon(\mathbf{u}(x), \mathbf{v}(y))$.

Using continuity of $\Upsilon$, boundedness of $\mathbf{v}^{1}\left(H_{Y}^{\kappa}\right), \mathbf{u}^{1}\left(H_{X}^{\varepsilon}\right)$, and [13, (2.34)], we obtain that

$$
\left.\mathrm{V}(\varepsilon, \delta)=\max _{b \in c l\left(\mathbf{v}^{1}\left(H_{Y}^{\kappa}\right), \tau_{\mathbb{R}}^{(l)}\right)} \min _{a \in c l\left(\mathbf{u}^{1}\left(H_{X}^{\varepsilon}\right), \tau_{\mathbb{R}}^{(k)}\right)} \Upsilon(a, b) \in \mathbb{R} \forall \varepsilon, \kappa \in\right] 0, \infty[
$$

where $\tau_{\mathbb{R}}^{(k)}$ and $\tau_{\mathbb{R}}^{(l)}$ are the topologies of coordinate-wise convergence in $\mathbb{R}^{k}$ and $\mathbb{R}^{l}$ respectively. Moreover, the asymptotic maximin as well-defined:

$$
\mathcal{V} \triangleq \max _{b \in \mathbb{G}_{2}} \min _{a \in \mathbb{G}_{1}} \Upsilon(a, b) \in \mathbb{R}
$$


where the following attraction sets (see $[2,(3.3 .10)]$ )

$$
\mathbb{G}_{1} \triangleq \bigcap_{\varepsilon \in] 0, \infty[} \operatorname{cl}\left(\mathbf{u}^{1}\left(H_{X}^{\varepsilon}\right), \tau_{\mathbb{R}}^{(k)}\right), \mathbb{G}_{2} \triangleq \bigcap_{\kappa \in] 0, \infty[} c l\left(\mathbf{v}^{1}\left(H_{Y}^{\kappa}\right), \tau_{\mathbb{R}}^{(l)}\right)
$$

are compacta. We now ready to state the specific version of [13, theorem 1$]$.

Theorem 1. The following approximation property of $\mathcal{V}$ holds:

$$
\forall \xi \in] 0, \infty\left[\exists \theta_{\xi} \in\right] 0, \infty[:|\mathcal{V}-\mathrm{V}(\varepsilon, \delta)|<\xi \forall \varepsilon \in] 0, \theta_{\xi}[\forall \kappa \in] 0, \theta_{\xi}[
$$

The extension of the original problem (2) is constructed in the following way: for each point of the sets $X$ and $Y$ we assign the Dirac measure supported at this point (see immersion operator $\Delta$ in $[3, \mathrm{p} \mathrm{1090]}$ and $[14,(4.4)]$ ). The closures of resulting sets w.r.t. *-weak topology coincide, respectively, with the set of all $\{0,1\}$-valued FAM on $\mathcal{L}_{X}$ and on $\mathcal{L}_{Y}$ (see $[3$, p. 1090] and $[14,(4.5)]$ ). We now introduce the specific version of sets $A$ and $B$ (see (1)):

$$
D_{X} \triangleq \bigcap_{\varepsilon \in] 0, \infty[} \operatorname{cl}\left(\left\{\delta_{x}^{X}: x \in H_{X}^{\varepsilon}\right\}, \tau_{\mathcal{L}_{X}}\right), D_{Y} \triangleq \bigcap_{\kappa \in] 0, \infty[} c l\left(\left\{\delta_{y}^{Y}: y \in H_{Y}^{\kappa}\right\}, \tau_{\mathcal{L}_{Y}}\right)
$$

where $\operatorname{cl}(Z, \tau)$ is the closure of $Z$ w.r.t. topology $\tau, \delta_{x}^{X}$ is the Dirac measure on $\mathcal{L}_{X}$ supported at $x, \delta_{y}^{Y}$ is the Dirac measure on $\mathcal{L}_{Y}$ supported at $y$, and $\tau_{\mathcal{L}_{X}}, \tau_{\mathcal{L}_{Y}}$ are *-weak topologies. From [11, proposition 2.2] it follows that sets $D_{X}, D_{Y}$ contains only purely FAM. Now we define the generalized payoff function $\tilde{\Upsilon}$ : $D_{X} \times D_{Y} \rightarrow \mathbb{R}$ by the rule

$$
\tilde{\Upsilon} \triangleq \Upsilon\left(\left(\int_{X} \mathbf{u}_{i} \mu(d x)\right)_{i \in \overline{1, k}},\left(\int_{Y} \mathbf{v}_{j} \nu(d y)\right)_{j \in \overline{1, l}}\right) \forall \mu \in D_{X} \forall \nu \in D_{Y}
$$

Using [13, proposition 5],(3), and (4), we obtain the next statement.

Theorem 2. The following representation of asymptotics of values of problems (2) holds: $\mathcal{V}=\max _{\nu \in D_{Y}} \min _{\mu \in D_{X}} \tilde{\Upsilon}(\mu, \nu)$.

It is shown that if we use the extension in the class of FAM, then it is possible to obtain representation of asymptotics of values of problems (2). Moreover, from Theorem 2 it follows that these asymptotics can be defined in terms of generalized elements. These elements are purely FAM.

Acknowledgements. The reported study was partially supported by RFBR (research projects No. 13-01-90414 Ukr_f_a, No. 13-01-00304 A). 


\section{References}

[1] J. Warga, Optimal Control of Differential and Functional Equations, Academic Press, New York, 1972.

[2] A.G. Chentsov, Asymptotic Attainability, Kluwer, Dordrecht, 1997.

[3] A.G. Chentsov, Finitely-additive measures and relaxations of abstract control problems, Journal of Mathematical Sciences, Vol. 133, 2 (2006).

[4] N.N. Krasovskii and A.I. Subbotin, Game-theoretical Control Problems, New York, Springer-Verlag, 1988.

[5] Y.V. Averboukh, Nash equilibrium for differential game and nonanticipative strategies technique [In Russian]. Matematicheskaya Teoriya Igr $i$ Ee Prilozheniya, 3 (2012), 3-20.

[6] D.V. Khlopin and A.G. Chentsov, On a control problem with incomplete information: quasistrategies and control procedures with a model. Differential Equations, 12 (2005), 1727-1742.

[7] A.P. Baklanov, On the representation of maximin of an impulse control problem [in Russian], Differential Equations and Control Processes, 3 (2012), 49-69.

[8] A.P. Baklanov and A.G. Chentsov, On question about extension of a game problem in the class of two-valued finitely-additive measures [in Russian], Tambov University Bulletin, 1 (2011), 15-37.

[9] A.G. Chentsov and Yu.V. Shapar, A game problem with approximate observation of constraints, Doklady Mathematics, 1 (2009), 497-502.

[10] A.P. Baklanov, A game problem with asymptotic impulse control [in Russian], Bulletin of Udmurt University. Mathematics., 3 (2011), 3-14.

[11] S.I. Morina and A.G. Chentsov, On a problem of asymptotic optimization [in Russian], Bulletin of Chelyabinsk State University. Mathematics, Mechanics, Computer Science, 2 (1994), 80-87.

[12] N. Bourbaki, General Topology, Hermann, Paris, 1940.

[13] A.G. Chentsov, About presentation of maximin in the game problem with constraints of asymptotic character [in Russian]. Bulletin of Udmurt University. Mathematics, 3 (2010), 104-119. 
[14] A.P. Baklanov and A.G. Chentsov, On question about extension of abstract attainability problems admitting discontinuous dependences, Functional Differential Equations, 12 (2010), 21-50.

\section{Received: October 3, 2013}

